\title{
EDITORIAL How RAGE turns in rage
}

\author{
CL Verweij \\ VU Medical Center, Department of Molecular Cell Biology, 1081 BT Amsterdam, The Netherlands
}

Genes and Immunity (2002) 3, 117-118. DOI: 10.1038/ sj/gene/6363865

The genetic background strongly contributes to susceptibility and clinical outcome of inflammatory diseases. The genetics of many of these diseases is complex, involving multiple genes. In the case of rheumatoid arthritis (RA), a chronic inflammatory joint disease, estimates suggest that at least 10 different genetic regions may be linked to susceptibility. ${ }^{1}$ Parametric linkage analysis suggests that the major histocompatibility complex (MHC) region may contribute up to $30 \%$ of overall inherited susceptibility in RA. $^{2}$ Good evidence exists that RA is associated with HLA-DRB1 alleles, in particular alleles that share a short amino acid sequence QKRAA, QRRAA or RRRAA at position 70-74 in the binding groove of the MHC molecule found in HLA-DR1 (HLA-DRB1*0101 and 0102), DR4 (HLA-DRB1*0401, 0404，0405), DR6 (HLA-DRB1* 1402) and DR10 (HLA-DRB1*1001) specificities named shared epitope alleles. ${ }^{3}$ Thus besides HLA, additional regions contribute to disease whereby differences in the constitution of these factors may relate to variability in clinical presentation with a spectrum ranging from mild to severe disease.

In this issue Hofmann et $a l^{4}$ provide evidence for a role of the gene encoding the receptor for advanced glycation end products (RAGE), implicated in the amplification of immune responses, as good candidate for a genetic factor that contributes to RA. ${ }^{4}$ Previous studies already suggested a pivotal role of RAGE activation in the pathogenesis of diabetic vascular disease and Alzheimer. ${ }^{5,6}$

RAGE is a multiligand member of the immunoglobulin superfamily of cell surface receptors that binds advanced glycation end products (AGE) - the products of nonenzymatic glycoxidation of protein/lipids-and amyloid$\beta$ peptide, a cleavage product of the $\beta$-amyloid precursor protein. ${ }^{5-7}$ Subsequent studies revealed that RAGE was also implicated as a receptor of proinflammatory ligands, the so-called extracellular newly identified RAGE binding proteins (EN-RAGEs) and related members of the S100/calgranulins family, that accumulate at sites of chronic inflammation. ${ }^{8}$ Signaling studies revealed that

Correspondence: $C L$ Verweij, PhD, VU Medical Center, Department of Molecular Cell Biology, 1081 BT Amsterdam, The Netherlands. E-mail: c.verweij.cell@med.vu.nl ligation of cell surface RAGE activates cell signaling pathways such as p21 Ras, ERK1/ERK2 kinases and NF-кB, thereby activating an inflammatory gene expression profile that typifies chronic inflammatory disorders. ${ }^{9}$ The EN-RAGEs S100A8 (myeloid related protein 8 (MRP)) and S100A9 (MRP14) are specifically secreted by monocytes upon interaction with inflammation activated endothelium. ${ }^{10}$ Hence, the subsequent interaction of the secreted EN-RAGEs with cell surface RAGE is expected to further amplify the inflammatory response and suggests a pivotal role for EN-RAGEs and RAGE in chronic cellular activation. The observation that the levels of MRP8 and 14 were elevated in juvenile RA and correlated with disease activity is in support for a role of EN-RAGEs in chronic inflammation. ${ }^{11}$ It is in this context that the authors performed studies in a collagen-induced murine arthritis model, which demonstrated that blockade of RAGE suppressed clinical, molecular and histological evidence of arthritis, thereby supporting a functional role for RAGE in arthritis. ${ }^{4}$

These findings made the RAGE gene an attractive candidate to study for the existence of allelic variations that might be important in the pathogenesis of inflammatory disease. The RAGE gene is located in the MHC locus on chromosome 6p21.3, which contains multiple genes involved in inflammatory and immune responses and is known for its multifold disease associations. Most interestingly, the search for heterogeneity within the RAGE gene uncovered several polymorphisms. ${ }^{12,13}$ However, since polymorphic genes within the MHC display a high degree of linkage disequilibrium, disease-associations found do not necessarily direct to a functional variation but could well be the consequence of a nearby diseasecausing allele. In fact, most disease-associated polymorphisms are functionally inert or lack information on the functional relevance.

For those reasons it is specially noteworthy that in the same paper Hofmann et $a l^{4}$ elegantly provide evidence for a functional difference between the naturally occuring RAGE protein variants, ie, the wild-type with a glycine at position 82 and the variant with a serine in that position. The studies revealed significant differences in (i) the $\mathrm{Kd}$ between RAGE $82 \mathrm{G}(\mathrm{Kd} \sim 122+/-31 \mathrm{nM})$ and RAGE $82 S$ (Kd 77+/-21 nM) receptors, (ii) the EN-RAGE induced phosphorylation of MEK1/2 and p44/42 MAP kinases and NF- $\mathrm{B}$ induction (RAGE $82 \mathrm{~S}>\mathrm{G}$ ), and (iii) EN-RAGE induced production of the pro-inflammatory 
cytokines TNF- $\alpha$ and IL- 6 , and the metalloproteinases (MMP) 3, 9 and 13 (RAGE 82S>G). These results demonstrate a significant functional difference in binding, signalling and gene expression between the allelic RAGE $82 \mathrm{~S}$ and $82 \mathrm{G}$ receptors. In a previous study Hudson et $\mathrm{al}^{12}$ reported on the functional relevance of RAGE promoter polymorphisms at position $-429,-374$ and -63 , suggesting that also genetically determined differences at the level of RAGE production may exist. ${ }^{12}$ Together these data suggest that polymorphism within the RAGE gene affect RAGE signalling and may influence disease susceptibility and/or outcome.

These observations raise the possibility that a predisposition to a vigorous RAGE responsiveness may be associated with chronic inflammatory diseases like RA and other autoimmune diseases. In the same study Hofman $e a^{4}{ }^{4}$ reported that the genetic variant that correlates with the expression of the more active RAGE $82 S$ receptor, confers increased susceptibility for the development of RA. However, the picture is more complicated as it seems due to the high degree of linkage disequilibrium within the MHC. Two major extensive HLA class II haplotypes associated with the 82S variant, HLA-DRB1* 0401-DQA1*0301-DQB1*0301 and HLA-DRB1*1501DQA1*0102-DQB1*0602, are described. ${ }^{13}$ When corrected for the contribution of HLA-DRB1*0401 in RA the association did not reach statistical significance. However, the inclusion of RAGE polymorphisms within HLA-DRB1* 0401 haplotypes could well affect the severity of disease. Assignment of functional RAGE gene variants that predict outcome will be helpful to stratify patients for clinical studies and tailor treatment. Therefore, further studies are necessary to explore the association of functional RAGE polymorphisms with outcome measures of laboratory parameters, such as TNF- $\alpha$, IL- 6 and MMPs, and clinical severity.

\section{References}

1 Cornelis F, Faure S, Martinez M et al. New susceptibility locus for rheumatoid arthritis suggested by a genome-wide linkage study. Proc Natl Acad Sci USA 1998; 95: 10746-10750.

2 Gregersen PK, Silver J, Winchester RJ. The shared epitope hypothesis. An approach to understanding the molecular genetics of susceptibility to rheumatoid arthritis. Arthritis Rheum 1987; 30: 1205-1213.

3 Wordsworth P, Bell J. Polygenic susceptibility in rheumatoid arthritis. Ann Rheum Dis 1991; 50: 343-346.

4 Hofmann MA, Drury S, Hudson BI et al. RAGE and arthritis: the G82S polymorphism amplifies the inflammatory response. Genes Immun 2002; 3.

5 Schmidt AM, Vianna M, Gerlach M et al. Isolation and characterization of two binding proteins for advanced glycosylation end products from bovine lung which are present on the endothelial cell surface. I Biol Chem 1992; 267: 14987-14997.

6 Yan SD, Chen X, Fu J et al. RAGE and amyloid-beta peptide neurotoxicity in Alzheimer's disease. Nature 1996; 382: 685-691.

7 Brownlee M. Advanced protein glycosylation in diabetes and aging. Annu Rev Med 1995; 46: 223-234.

8 Hofmann MA, Drury S, Fu C et al. RAGE mediates a novel proinflammatory axis: a central cell surface receptor for S100/ calgranulin polypeptides. Cell 1999; 97: 889-901.

9 Kislinger T, Fu C, Huber B et al. N(epsilon)(carboxymethyl)lysine adducts of proteins are ligands for receptor for advanced glycation end products that activate cell signaling pathways and modulate gene expression. J Biol Chem 1999; 274: 31740-31749.

10 Rammes A, Roth J, Goebeler $\mathrm{M}$ et al. Myeloid-related protein (MRP) 8 and MRP14, calcium-binding proteins of the S100 family, are secreted by activated monocytes via a novel, tubulin-dependent pathway J Biol Chem 1997; 272: 9496-9502.

11 Frosch M, Strey A, Vogl T et al. Myeloid-related proteins 8 and 14 are specifically secreted during interaction of phagocytes and activated endothelium and are useful markers for monitoring disease activity in pauciarticular-onset juvenile rheumatoid arthritis. Arthritis Rheum 2000; 43: 628-637.

12 Hudson BI, Stickland MH, Grant PJ. Identification of polymorphisms in the receptor for advanced glycation end products (RAGE) gene: prevalence in type 2 diabetes and ethnic groups. Diabetes 1998; 47: 1155-1157.

13 Prevost G, Fajardy I, Fontaine P, Danze PM, Besmond C. Human RAGE GLY82SER dimorphism and HLA class II DRB1DQA1-DQB1 haplotypes in type 1 diabetes. Eur J Immunogenet 1999; 26: 343-348. 